\title{
Direct assessment of p-n junction in single GaN nanowires by Kelvin Probe Force Microscopy
}

\author{
Albert Minj ${ }^{1,2}$, Ana Cros ${ }^{1, *}$, Thomas Auzelle ${ }^{3,4}$, Julien Pernot ${ }^{5,6,7}$ and Bruno Daudin ${ }^{3,4}$ \\ 1) Institute of Materials Science (ICMUV), Universidad de Valencia, P.O. Box 22085, E-46071, Valencia, \\ Spain \\ 2) CIMAP, UMR 6252, ENSICAEN, 6 Bd Maréchal Juin, 14050 Caen Cedex 4, France \\ 3) Univ. Grenoble Alpes, INAC-SP2M, F-38000 Grenoble, France \\ 4) CEA, INAC-PHELIQS , «Nanophysique et semiconducteurs group», F-38000 Grenoble, France \\ 5) Univ. Grenoble Alpes, Inst NEEL, F-38042 Grenoble, France \\ 6) CNRS, Inst NEEL, F-38042 Grenoble, France \\ 7)Institut Universitaire de France, 103 boulevard Saint-Michel, F-75005 Paris, France
}

*E-mail: Ana.Cros@uv.es

\begin{abstract}
Making use of Kelvin probe force microscopy, in dark and under ultraviolet illumination, we study the characteristics of $p-n$ junctions formed along the axis of self-organized GaN nanowires (NWs). We map the contact potential difference of the single NW p-n junctions to locate the space charge region and directly measure the depletion width and the junction voltage. Simulations indicate a shrinkage of the built-in potential for NWs with small diameter due to surface band bending, in qualitative agreement with the measurements. The photovoltage of the NW/substrate contact is studied by analysing the response of NW segments with p- and n-type doping under illumination. Our results show that the shifts of the Fermi levels, and not the changes in surface band bending, are the most important effects under above band-gap illumination. The quantitative electrical information obtained here is important for the use of NW p-n junctions as photovoltaic or rectifying devices at the nanoscale, and is especially relevant since the technique does not require the formation of ohmic contacts to the NW junction.
\end{abstract}




\section{Introduction}

In the last few years, research interest in semiconductor nanowires (NWs) has experienced a rapid increase. This is especially true for III-nitrides, a material system where the presence of a high density of structural defects in epitaxial layers has often prevented the fine control of nanoscale material parameters for the development of efficient devices [1]. These NWs present clear structural advantages over their two-dimensional counterparts, but their fundamental characterization requires the assessment of single object properties, a task that often constitutes a difficult enterprise. In particular, their electrical characterization needs NW manipulation and the formation of electrical contacts at the nanometer scale, technologically demanding and hard to control. Activities that are common in two dimensional layers or conventional devices, such as the characterization of free carrier density, the type of carrier and the shape and extension of the depletion layers, constitute a crucial issue for the assessment of NW based p-n junctions or gated transistors. For the determination of carrier density different optical techniques not requiring electrical contacts have been proposed, such as Raman scattering [2] or photoluminescence [3], but their spatial resolution is diffraction limited. Characterization of $\mathrm{p}-\mathrm{n}$ junctions and devices by means of electron beam induced current measurements is a good alternative [4] in terms of high resolution and statistics, although the fact that this technique makes use of high-energy electron beams could affect dopant activation. Alternatively, scanning probe microscopy (SPM) techniques have proved to be adequate for the electrical and structural characterization of NWs [5]. With this respect, a contact-less technique such as Kelvin probe force microscopy (KPFM) has already allowed the observation of material and doping contrast in GaAs-based NWs with nanometer resolution and without the need of electrical contacts [6], providing direct evidence of space charge regions in InP and InAs NWs and their heterostructures [7]. It is also suitable for the study of biased p-n Si NW junctions and the determination of contact resistance of the order of $\mu \Omega \mathrm{cm}^{-2}[8]$. More generally, KPFM has also been used to assess the effect of dopants on the electronic potential of thin planar Si p-n junctions, 
including the effect of illumination $[9,10]$. However, the technologically relevant material system of nitride semiconductor NWs has seldom been addressed by this technique. Only recently, Imtiaz and collaborators [11] have reported the characterization of a p-n junction formed on a GaN NW by means of a related SPM technique, namely scanning microwave microscopy. We demonstrate here how electrical scanning probe techniques operating at room temperature and ambient conditions can provide a deeper understanding of device performance and guide device optimization without the need of nanoscale electrical contacts, thus enabling the study of shorter NWs (1-2 $\mu \mathrm{m}$ long). For this purpose, we use KPFM and light assisted KPFM under ambient conditions to study the electrical characteristics of single GaN NWs consisting of Si and Mg doped sections that form a p-n junction. With this study we are not only able to locate the p-n junction, but also to i) directly measure the depletion width, ii) investigate the junction potential, iii) determine the nature of doping and iv) study the photo-response of the junction and the NW/substrate contact. The experimental results are compared with simulations of the electronic structure of the NW performed with NextNano.

\section{Results and discussion}

The GaN p-n junction NWs studied in this work were grown by molecular beam epitaxy (MBE. See the Experimental Section), and consist of self-organized NWs with a Si doped section followed by a $\mathrm{Mg}$ doped section. Scanning electron micrographs (SEM, top and side views) of the as-grown GaN p-n NW junctions are shown in Figure 1 for two regions of the sample with small [a) and b)] and large [c) and d)] NWs. These two regions are related to the temperature gradient along the radius of the wafer, which results in an increasing density and widening of the NWs for lower temperature [12]. From the top views a) and c), which reflect the $c$-plane surface of the p-type region, an average diameter of 90 and $150 \mathrm{~nm}$ is measured, respectively. The base n-type region, measured from side views b) and d) has a narrower diameter (60 and $90 \mathrm{~nm}$, respectively). The wider top 
reflects an increased radial growth rate induced by Mg-incorporation. The diameter enlargement and the SEM contrast, obtained using an in-lens detector, are more evident in d) and roughly provide the location of the junction in the $\sim 1.5$ to $2 \mu \mathrm{m}$ long NWs, but assessment of the existence of a p-n junction requires additional electrical characterization. For this purpose, NWs were mechanically dispersed on highly oriented pyrolytic-graphite (HOPG) and on Au-coated $\mathrm{Si}$ substrates. Both substrates yielded similar results, but $\mathrm{V}_{\mathrm{CPD}}$ of NWs on HOPG was more reproducible, with better resolution and stable surface potential values. This is ascribed to the smoothness and softness of the surface combined with its hydrophobic nature, which minimizes the influence of the surface water layer present under atmospheric conditions. Furthermore, since the work function of HOPG in air is lower than that of gold (4.5 eV vs. $4.8 \mathrm{eV}$ [13]), it is expected to result in a Schottky contact with a smaller barrier for GaN NWs, characterized by an electron affinity of $4.1 \mathrm{eV}$ [14] and a strong Fermi-level pinning arising from surface states [15]. Besides its electrical properties, HOPG also constitutes an ideal platform for separating NW bunches into individual NWs by AFM nano-manipulation without destroying the tip [16].

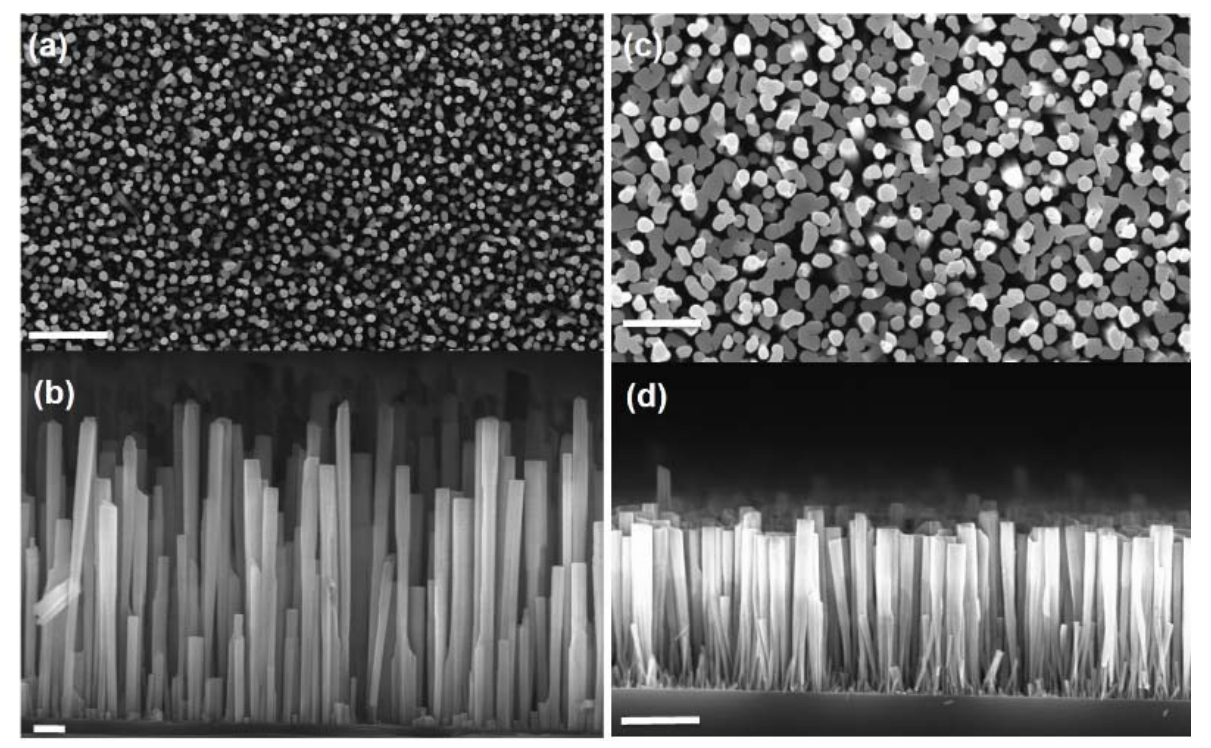

Figure 1. SEM micrographs of the as-grown p-n GaN NWs illustrating two different regions of the sample. The scale bar represents $1 \mu \mathrm{m}$ in a), c) and d), and $200 \mathrm{~nm}$ in b). 


\subsection{Junction voltage and space charge region in dark. Experiments and simulation}

We analyze the characteristics of several NW p-n junctions dispersed on HOPG. Complementary results obtained for NWs dispersed on gold are given in the Supporting Information. AFM topography and surface potential scans (KPFM image) of various single NW p-n junctions originating from the region of the sample depicted in Figure 1a and 1b are shown in Figure 2, together with the corresponding profiles taken along the marked lines. The quantity measured by KPFM, that is, the difference between the work function of the tip $\phi_{\text {ip }}$ and of the sample surface, $\phi_{\mathrm{s}}$, corresponds to the $\mathrm{V}_{\mathrm{CPD}} . \phi_{\mathrm{s}}$ is evaluated by taking into account the Fermi energy with respect to the bulk conduction band $\left(\mathrm{E}_{\mathrm{C}}-\mathrm{E}_{\mathrm{F}}\right)$, the surface band bending $\left(\phi_{\mathrm{p}}<0\right.$ for $\mathrm{p}$-type and $\phi_{\mathrm{n}}>0$ for $\mathrm{n}$ type), and the electron affinity $\chi$, and is given by $e \cdot V_{C P D}=\phi_{\text {tip }}-\left(\chi+E_{C}-E_{F}+\phi_{\mathrm{n}, \mathrm{p}}\right)$. These quantities are schematized in Figure 7. 

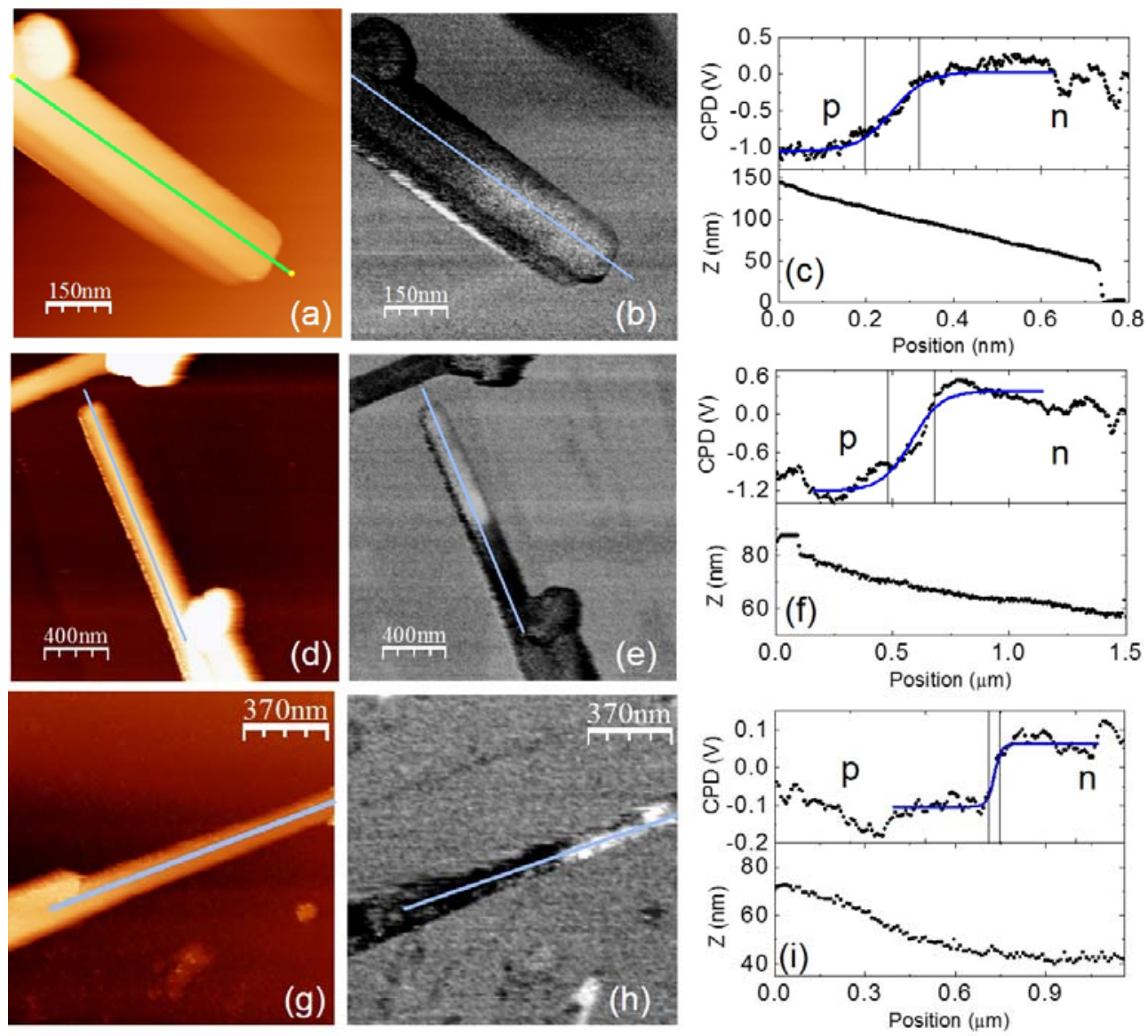

Figure 2. AFM topography a),d),g) and corresponding KPFM images b),e),h) of three characteristic p-n junction NWs. $V_{C P D}$ of HOPG is taken as reference and gray scale spans from -1.1 to $1.2 \mathrm{~V}$ in $\mathrm{b}$ ) and e) and from -0.11 to $0.12 \mathrm{~V}$ in $\mathrm{h})$. c),f),i) Topography and $\mathrm{V}_{\text {CPD }}$ profiles along the marked lines. The blue line is a fit to a sigmoid curve.

In the KPFM images [b), e) and h)] we observe a strong contrast between the p-type (dark) and the n-type (bright) regions, correlated with the thicker and narrower sides of the NWs, respectively. $\mathrm{V}_{\mathrm{CPD}}$ values increase from the $\mathrm{p}$ - to the $\mathrm{n}$-type sides in accordance with the fact that in our experiment the compensating DC bias that provides $\mathrm{V}_{\mathrm{CPD}}$ is applied to the tip [17]. This contrast is an evidence of the effective charge separation in the space charge region due to the formation of the junction. Only in the absence of surface band bending the difference $\Delta \mathrm{V}_{\mathrm{CPD}}$ between the $\mathrm{n}$ and $\mathrm{p}$ 
sides would correspond to the built-in potential, which for large doping is expected to be of the order of the band gap. Indeed, due to the presence of surface states and the associated band bending an upward surface band bending is expected at the m-plane surface of the n-type side [18], while for p-type GaN the band bending is expected to be downwards, as shown by Long et al. for c-plane $\mathrm{GaN}$ [19]. As a result, the measured $\Delta \mathrm{V}_{\mathrm{CPD}}$ gives a lower limit to the junction built-in potential. Actually, the variability observed in the characteristics of the NW p-n junction is large: $\Delta \mathrm{V}_{\mathrm{CPD}}$ ranges from $0.17 \mathrm{~V}$ (Figure 2i) to $1.59 \mathrm{~V}$ (Figure 2f). Possible origins of these changes are discussed later, in view of the results of the simulations. Our measurements also provide the extension of the space charge region (SCR) that has been obtained from the experimental data by a fit of the $V_{C P D}$ profile with a sigmoid curve. The results of the fit, together with the value of $\Delta V_{C P D}$ and the NW diameter of the $\mathrm{n}$ side are given in Table I. The diameter is determined from the $z$ value of the topography, since this measure is not affected by the size of the tip. It takes its minimum at the NW edge in the n-type side, increasing by a few nanometers towards the p-n junction. Special care was taken when determining the diameter of the NW shown in Figure 2a, since the $\mathrm{n}$ side of this NW rests on the HOPG surface, but its $p$ side is on a bunch of NWs. Consequently, the large diameter enlargement displayed in Figure 2c when moving from the $\mathrm{n}$ towards the $\mathrm{p}$ side does not reflect the real NW diameter, which is in the range from 55 to $65 \mathrm{~nm}$. In the NWs investigated, SCR varies from 40 to $200 \mathrm{~nm}$, the larger values corresponding to larger $\Delta \mathrm{V}_{\mathrm{CPD}}$ and $\mathrm{NW}$ diameters. 
Table I. Electrical and structural characteristics of the measured NW p-n junctions. The letters labeling the NWs refer to Figure 2.

\begin{tabular}{|c|c|c|c|}
\hline NW & Diameter n-side (nm) & $\Delta \mathrm{V}_{\mathrm{CPD}}(\mathrm{V})$ & $\mathrm{SCR}(\mathrm{nm})$ \\
\hline $\mathrm{g}$ & $40-43$ & 0.17 & 40 \\
\hline $\mathrm{a}$ & $55-65$ & 1.07 & 124 \\
\hline $\mathrm{d}$ & $60-65$ & 1.59 & 200 \\
\hline
\end{tabular}

3D Finite element simulations (nextnano3 software) have been performed in order to describe quantitatively the electrostatic properties of the p-n junction measured by KPFM experiments. Similar doping levels $\left(\mathrm{N}_{\mathrm{D}}=\mathrm{N}_{\mathrm{A}}=3.8 \times 10^{18} \mathrm{~cm}^{-3}\right)$ and fixed surface charges $\left(\mathrm{N}_{\text {surf }(-)}=\mathrm{N}_{\text {surf (+) }}=3 \times 10^{12}\right.$ $\mathrm{cm}^{-2}$ ) have been assumed on each side of the $\mathrm{p}-\mathrm{n}$ junction. The fixed surface charges are compensating negatively charged acceptor states in the n-type region and positively charged donor states in the p-type region. The results are shown on Figure 3. Figure 3a exhibits the electric potential of a $x y$ cross-section $(z=0$, i. e. the $\mathrm{NW}$ core) of the $\mathrm{NW}$, which corresponds to a $2 \mathrm{D}$ slice at the core, along the NW axis. As usual, the electrical potential gradient is maximum at the p-n junction location due to charge depletion near the p-n interface. However, the axial potential drop is lower near the NW surfaces due to the near surface radial depletion induced by fixed surface charges. Fixed surface charges induce a near surface band bending along the radial direction, as shown in Figure $3 \mathrm{~b}$ and Figure 3d. Figure $3 \mathrm{~b}$ shows the bottom of the conduction band and the top of the valence band (solid lines) versus $y$-position in the neutral n-type region. The dashed line corresponds to the Fermi level position. In the n-type region, a near surface upward band bending appears and creates a larger gap between the conduction band and the Fermi level compared to the core of the NW. In the p-type region, a downward band bending is observed. The energy barrier 
between the surface and neutral region of each side ( $\mathrm{n}$ and $\mathrm{p}$ region) is around $0.5 \mathrm{eV}$. This effect, combined to the low screening of the potential at the air/GaN interface, induces a larger built-in potential of the p-n junction at the core of the $\mathrm{NW}(\approx 3 \mathrm{eV})$ than at its surface $(\approx 2 \mathrm{eV})$. As shown on Figure 3c, the built-in potential at the core of the NW in the neutral region (black line) is $1 \mathrm{eV}$ larger than the one at the surface of the NW (red line). The space charge region extends symmetrically from the p-n junction along the $x$-axis. The total space charge region width is around $100 \mathrm{~nm}$ at the core and at the surface of the wire, in reasonable agreement with the KPFM data.

The decrease of the NW diameter induces an additional shrinking of the built-in potential. Indeed, as shown by simulation, the near surface space charge region induced by the band bending is about 15-20 $\mathrm{nm}$ on each side of the surface. This depleted area can be larger than the NW radius. In that case, the fixed surface charges completely deplete the dopants of the wire at each side of the junction and no neutral region is observed anymore in the wire core. As a consequence, the resulting built-in potential of the junction (Fermi level difference between n-type and p-type regions) shrinks for low NW diameters. Even if the calculated built-in potential and space charge region width are in reasonable agreement with the KPFM data, this simulation cannot give a quantitative fitting of the experiments because of the lack of accurate input parameter values like doping levels at each side of the junction, interface state density at each side of the junction and energetic position in the band gap of the fixed charges at the origin of the Fermi level pinning [20]. However, the simulation explains two major effects observed from KPFM data: i) the built-in potential values measured at the surface of the p-n junction of the GaN NW (maximum $1.59 \mathrm{eV}$ ) are lower than in bulk p-n junctions $(\approx 3 \mathrm{eV})$, ii) the built-in potential decreases versus the NW diameter. 

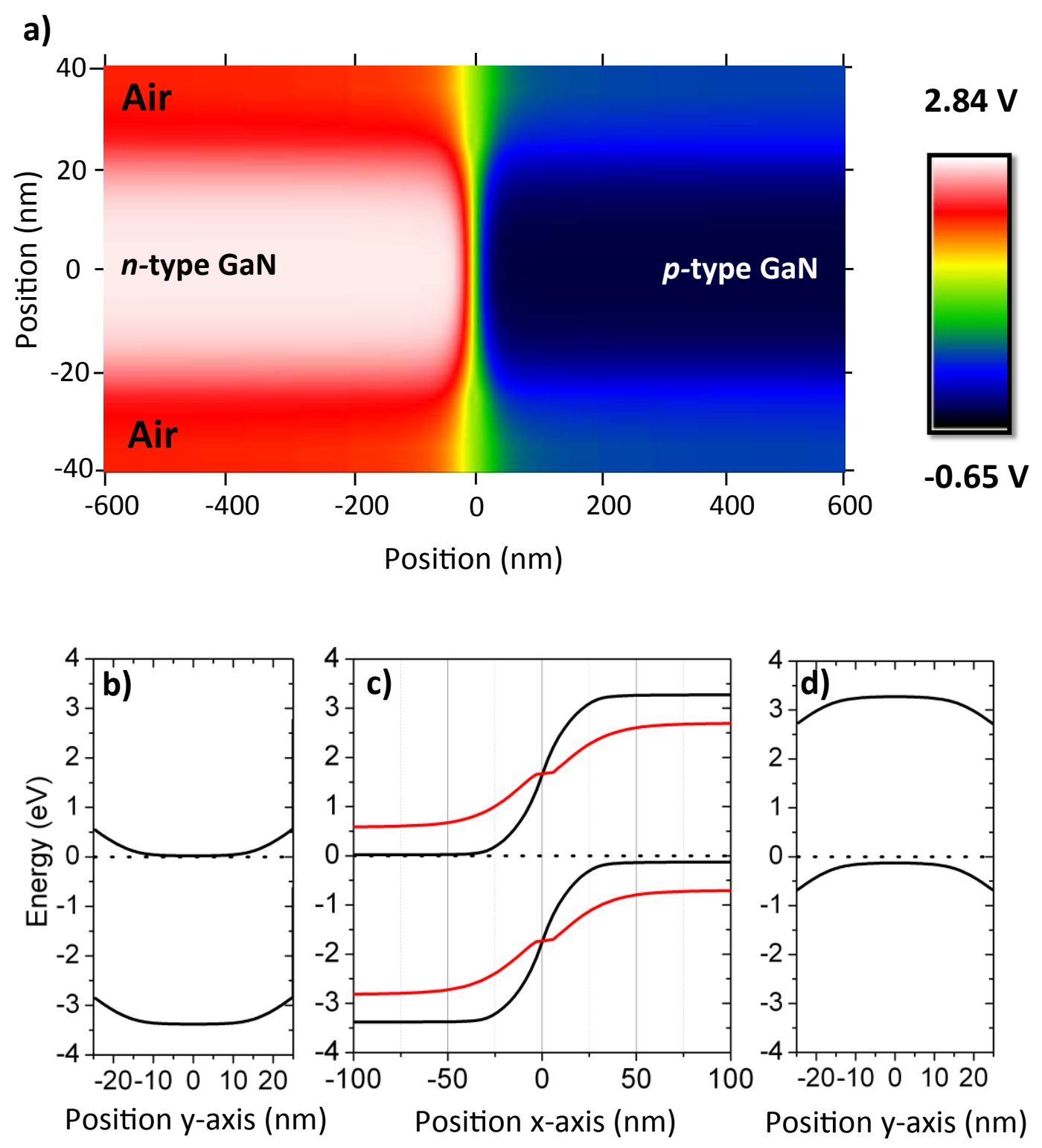

Figure 3. 3D finite element calculations of a $50 \mathrm{~nm}$ diameter $\mathrm{GaN}$ NW using similar doping levels $\left(\mathrm{N}_{\mathrm{D}}=\mathrm{N}_{\mathrm{A}}=3.8 \times 10^{18} \mathrm{~cm}^{-3}\right)$ and fixed surface charges $\left(\mathrm{N}_{\text {surf (-) }}=\mathrm{N}_{\text {surf }(+)}=3 \times 10^{12} \mathrm{~cm}^{-2}\right)$ on each side of the p-n junction. a) $x y$ cross section $(z=0)$ of the electrical potential at the vicinity of the $p-n$ junction. b) Conduction band minimum and valence band maximum (solid lines) versus $y$-position at $x=-400 \mathrm{~nm}$ (neutral $\mathrm{n}$-type side). The dashed line corresponds to the Fermi level position. c) Same as in b) but versus $x$-position at $y=0 \mathrm{~nm}$ (black line: core of the NW) and at $\mathrm{y}=25 \mathrm{~nm}$ (red line: surface of the NW). d) Same as b) but at $x=400 \mathrm{~nm}$ (neutral p-type side). 


\subsection{Response to illumination. p-n junction and NW/HOPG contact}

The response of a p-n junction (NW d) of Figure 2) under UV illumination was also studied and is shown in Figure 4, where HOPG is taken as reference for the $\mathrm{V}_{\mathrm{CPD}}$ values. With this purpose, the NWs were illuminated with a $100 \mathrm{~W}$ mercury lamp, with a measured light power on the sample of $40 \mathrm{~mW}$ on a spot of around $1 \mathrm{~cm}^{2}$. Under illumination, the $\mathrm{V}_{\mathrm{CPD}}$ difference between the $\mathrm{n}$ and $\mathrm{p}$ sides is strongly reduced, as expected from the photovoltaic effect: above band-gap illumination generates electron hole pairs that are separated by the built-in electric field at the depletion region and move towards the $\mathrm{n}$ and $\mathrm{p}$ side, respectively. This results in the generation of a voltage across the junction or the junction becoming forward biased, with the consequent reduction in built-in potential mainly due to the shift of the Fermi levels of both sides relative to each other. The $\mathrm{V}_{\mathrm{CPD}}$ at the $\mathrm{p}$ side increases by $0.73 \mathrm{~V}$, while that of the $\mathrm{n}$ side decreases by $0.47 \mathrm{~V}$. The observed $\mathrm{V}_{\mathrm{CPD}}$ changes demonstrate the formation of quasi-Fermi levels, revealing an effective induced voltage of $\sim 1.2 \mathrm{~V}$ assigned to the photovoltaic effect.
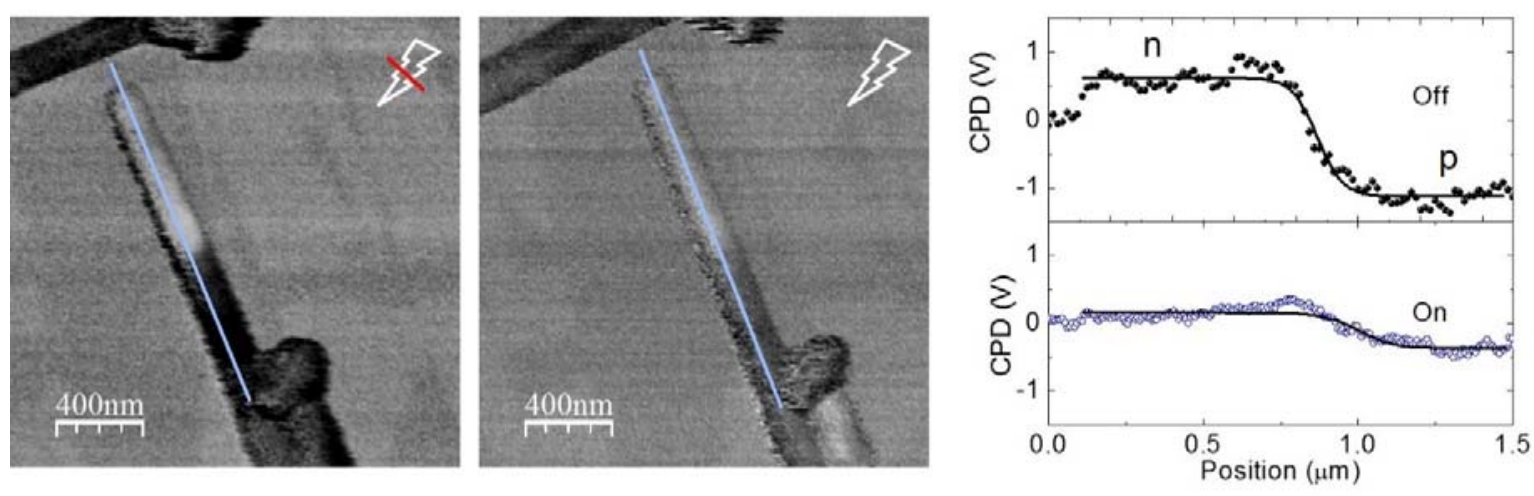

Figure 4. NW $V_{C P D} a$ ) at dark and b) under illumination. c) and d) $V_{C P D}$ profiles along the NW axis with light off and on, respectively. The gray scales span from -1.1 to $1.2 \mathrm{~V}$. The lines are fits to a sigmoid curve. 
In order to measure the evolution of $\mathrm{V}_{\mathrm{CPD}}$ with illumination the scan direction was aligned along the NW axis, which allows the simultaneous study of the $\mathrm{p}$ and $\mathrm{n}$ sides. Light was switched on for 28 minutes and then switched off again. The evolution of $\mathrm{V}_{\mathrm{CPD}}$ with light of the $\mathrm{n}$ and $\mathrm{p}$ sides is shown in Figure 5. Upon illumination the photo-induced changes take place very quickly and are followed by stable $\mathrm{V}_{\mathrm{CPD}}$ values. This behavior is in contrast to that observed on freshly etched c-plane GaN, where the quick change induced by carrier photoexcitation and reorganization is followed by slower changes ascribed to chemisorption of oxygen [21]. The constant $V_{C P D}$ observed during illumination reveals the oxidation of the NW surface. After illumination is switched off the $\mathrm{p}$ and $\mathrm{n}$ sides do not recover immediately their original potential value. A similar behavior has been analyzed by Foussekis et al. [22] in not-intentionally doped bulk GaN, and attributed to a slow electron transfer process between the chemisorbed oxygen and the $\mathrm{GaN}$ surface by tunneling through the thin surface oxide layer.
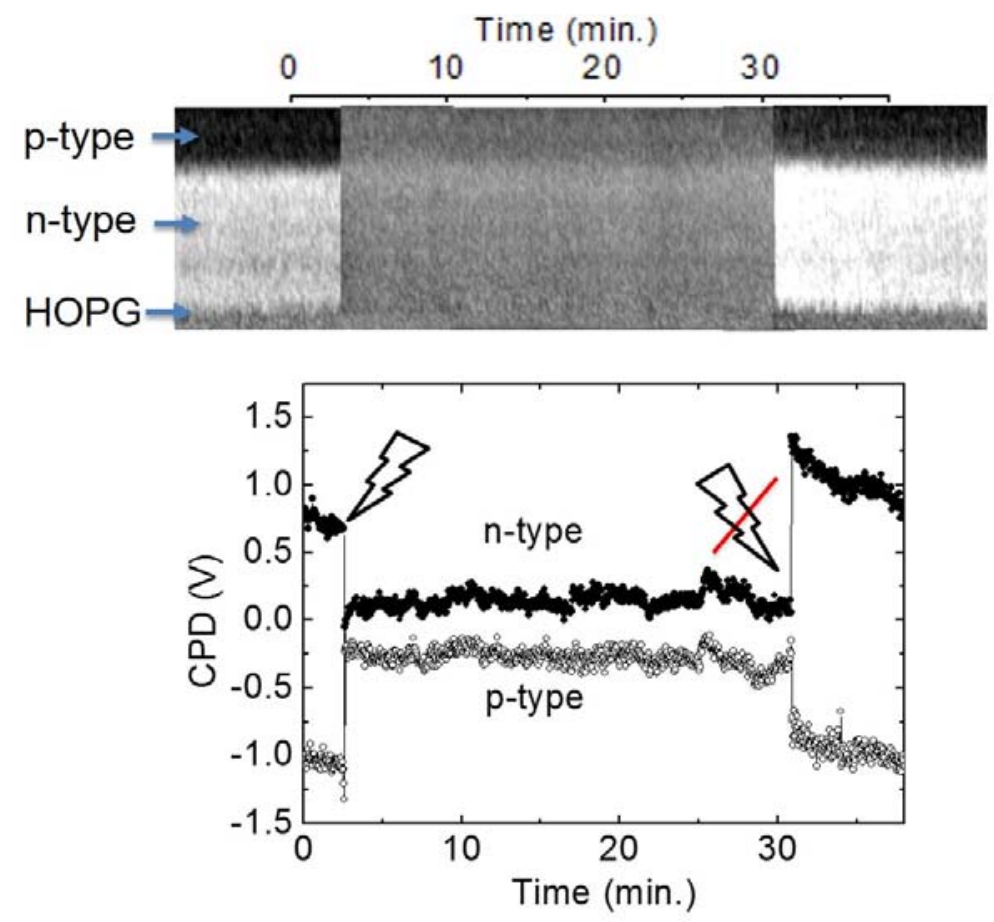

Figure 5. Effect of illumination on $\mathrm{V}_{\mathrm{CPD}}$ at the $\mathrm{p}$ and $\mathrm{n}$ sides. $\mathrm{V}_{\mathrm{CPD}}$ of the HOPG substrate is taken as reference. 
In a follow-up work Foussekis et al. [23] also put in evidence an anomalous offset in $V_{\text {CPD }}$ under illumination of the contacts. This opens up the question of the influence of the contact between the GaN NW and the HOPG substrate on the measured photo-induced voltage, a feature that will be experimentally addressed in a next stage.

For this purpose, the electrical characteristics of NWs that consist either of only p-type or only ntype sections have been studied. These NWs are segments that broke during the NW mechanical detachment. The AFM topography of the two pieces studied are shown in panel c) of Figure 6 . The larger piece stems from the Mg-doped NW top and consists of several NWs in a bunch. The NW at the top of the bunch has a diameter of $100 \mathrm{~nm}$. We also studied a NW stemming from the Si-doped bottom. It corresponds to the thinner NW segment, located at the right side of the picture, with a diameter of $25 \mathrm{~nm}$. As seen in Figure 6a, the two NWs and the HOPG substrate present characteristic $V_{C P D}$ values. For the graphite substrate $V_{C P D}$ is almost zero, while for the thinner NW, it is slightly higher (appearing brighter), with a mean $\mathrm{V}_{\mathrm{CPD}}$ value of $60 \mathrm{mV}$. In contrast, the thicker NW appears very dark in the image with much smaller $V_{\text {CPD }}$ mean values of $-440 \mathrm{mV}$. Statistics of the $V_{\text {CPD }}$ image of the two NWs and the HOPG substrate are shown in panel d), fitted to Gaussian curves. The potential values of the substrate spread with a full width at half maximum (FWHM) of only $30 \mathrm{mV}$, the resolution of our AFM system and could be taken as the resolution limit of our measurements due to the high homogeneity of these kind of substrates. For the NWs the FWHM is considerably larger, around $150 \mathrm{mV}$, but the values of both populations are well separated and can be clearly distinguished. Since both expose the same m-plane to the AFM tip, the large differences in $\mathrm{V}_{\mathrm{CPD}}$ are ascribed to doping, that introduces changes in surface band bending and Fermi energy. We can estimate the effect of surface band bending on the n-type NW by taking into account the results of the simulation, where the surface barrier for a NW with a diameter of $50 \mathrm{~nm}$ is determined to be $0.5 \mathrm{eV}$. As commented before, when the surface depleted area is larger than the NW radius no neutral region exists in the wire core, and the surface band bending is effectively reduced. Based on 
the work of Stoica et al. [24], we estimate the band bending to be around $0.25 \mathrm{eV}$ for a NW with 25 $\mathrm{nm}$ diameter provided the donor density remains of the order of $3.8 \times 10^{18} \mathrm{~cm}^{-3}$. The situation for the p-type NW is more difficult to analyze, since studies on the band bending of m-polar surfaces of $p$ type GaN are scarce [25]. For the c-plane T. Hashizume [26] and S. Barbet et al. [27] report on larger downward band-bending, of the order of 1.2 to $1.6 \mathrm{eV}$, with hole densities of the order of $7 \times 10^{16} \mathrm{~cm}^{-3}$. Altogether, given its large diameter we expect a larger band bending for the p-type NW than for the n-type NW.
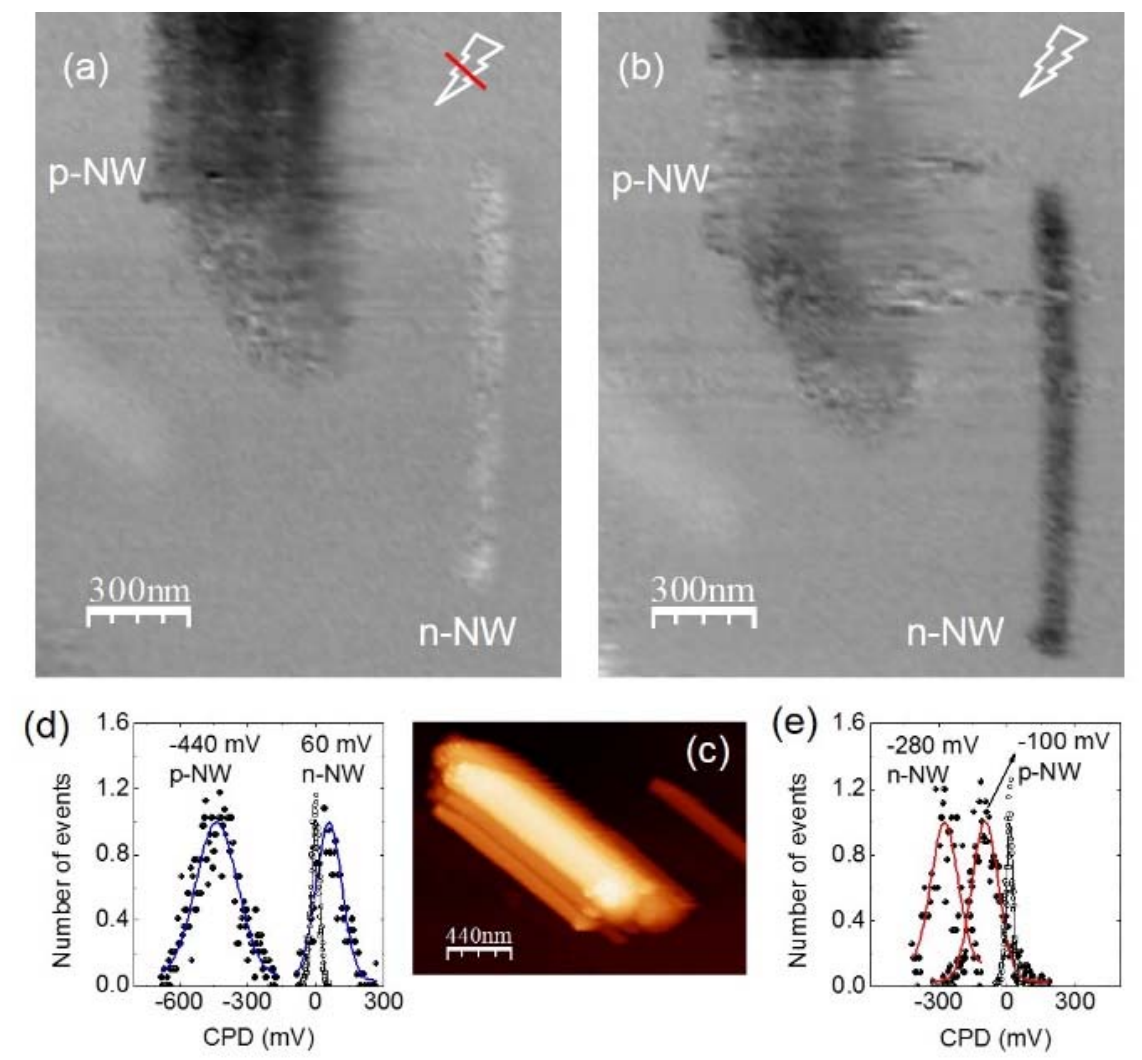

Figure 6. KPFM study of p-and n-type NWs. (a) KPFM in dark. b) KPFM under UV illumination. Gray scale spans -0.7 to $0.5 \mathrm{~V}$ in both pictures. c) Topography of the two NW segments. Color scale spans 0 to $140 \mathrm{~nm}$. d) CPD statistics of the p and n-type NWs (full dots) and the HOPG substrate (open dots) in dark. e) Same as in d), but under UV illumination. 
(a)

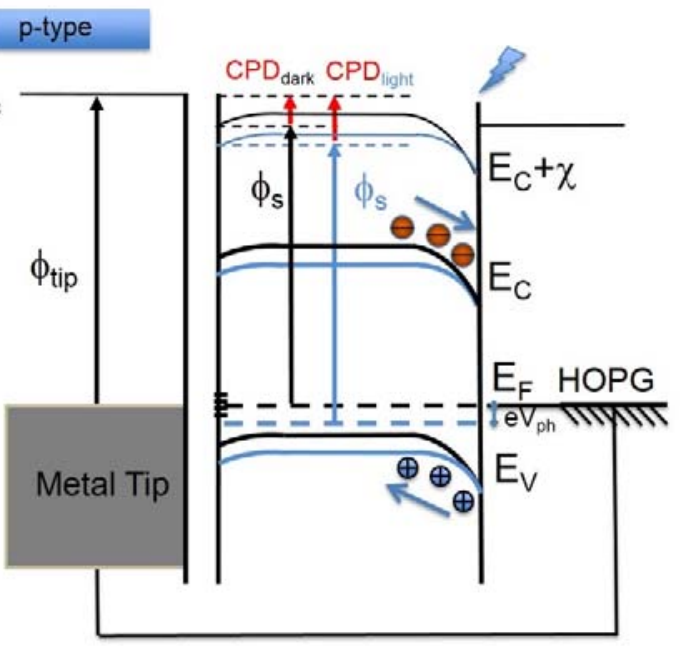

(b) n-type

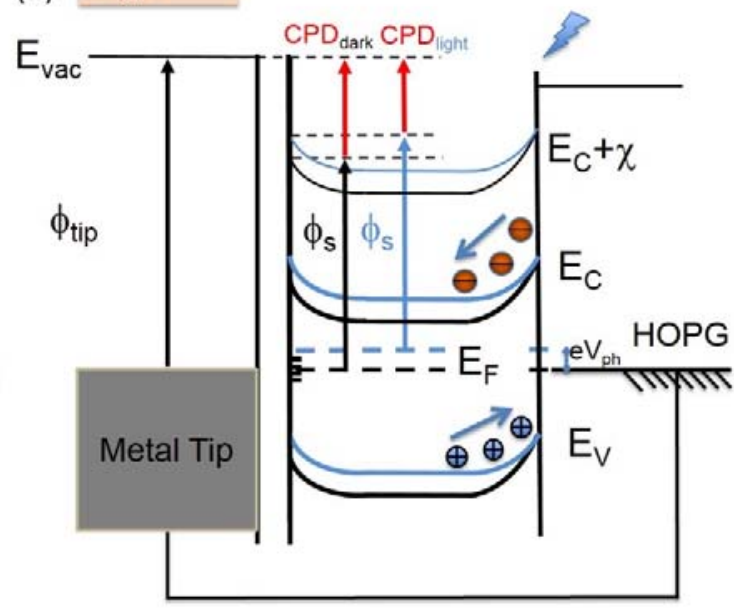

Figure 7. Schematic of the band structure of a) p-and b) n-type GaN at the m-plane surface. Detail of the band structure of the Schottky contact formed between the NW and the HOPG substrate is also given. The main changes introduced upon illumination are indicated in blue.

A schematic of the energy band diagrams relevant for KPFM measurements on the NW m plane is shown in Figure 7, where the grounded HOPG substrate is also included. The $V_{\text {CPD }}$ measured is depicted as a red arrow. $\phi_{\mathrm{s}}$ is evaluated by taking into account the Fermi energy with respect to the conduction band $\left(\mathrm{E}_{\mathrm{C}}-\mathrm{E}_{\mathrm{F}}\right)$, the surface band bending $\left(\phi_{\mathrm{p}}\right.$ for $\mathrm{p}$-type and $\phi_{\mathrm{n}}$ for n-type), and the electron affinity $\chi$ leading to the determination of $e \cdot V_{C P D}=\phi_{\text {tip }}-\left(\chi+E_{C}-E_{F}+\phi_{\mathrm{n}, \mathrm{p}}\right)$. In this scheme, panel a) refers to p-type and panel b) to n-type NWs. The fact that the Fermi energy is closer to the valence band in the p-type NW than in the n-type NW results in decreased $\mathrm{V}_{\mathrm{CPD}}$ values, as pointed out by the red arrow and as observed in the experiments. Importantly, the change in surface band bending from upward to downward would result in increased $\mathrm{V}_{\mathrm{CPD}}$ values, contrary to the experimental observation, which implies that the shift of the Fermi level is indeed the dominant effect, leading to a $\mathrm{V}_{\mathrm{CPD}}$ difference of $500 \mathrm{mV}$ between n-doped and $\mathrm{p}$-doped NWs, as shown in Figure 6d. 
Similar to the case of the p-n junction analyzed above, more insight into the band structure of the NWs and its interaction with the HOPG substrate can be obtained by analyzing the changes in $\mathrm{V}_{\mathrm{CPD}}$ under UV illumination. Figure 6, panels b) and e), show the new values of $\mathrm{V}_{\mathrm{CPD}}$ obtained after the UV light has been switched on. The effect of light can be directly observed in the p-type NW, since the first few lines (from the top of the image) of the scan were acquired with the light off. While there is no noticeable change in the HOPG substrate under illumination (just a small $12 \mathrm{mV}$ increase in $\mathrm{V}_{\mathrm{CPD}}$ ), the p-type $\mathrm{NW}$ appears bright (increase in $\mathrm{V}_{\mathrm{CPD}}$ ) and the n-type $\mathrm{NW}$ dark (decrease in $\mathrm{V}_{\mathrm{CPD}}$ ), opposite to what we observed when the light is off. The mean $\mathrm{V}_{\mathrm{CPD}}$ values under illumination are shown in panel e). The $\mathrm{V}_{\mathrm{CPD}}$ shift is the same for both $\mathrm{NWs}, 340 \mathrm{mV}$, but in opposite directions. The expected decrease of the surface band bending due to the neutralization of the surface states by the photogenerated carriers cannot explain the experimental results, since it would imply an opposite $\mathrm{V}_{\text {CPD }}$ change, namely a decrease for the p-type NW and an increase for the n-type NW $[19,28]$, contrary to our observations.

Alternatively, the shift in $\mathrm{V}_{\mathrm{CPD}}$ induced by UV illumination could be accounted for by considering the Schottky nature of the NW/HOPG contact. As schematized in the right part of Figure $7 \mathrm{a}$ and $7 \mathrm{~b}$, under illumination the carriers photogenerated near the junction separate at the Schottky barrier. In the case of n-type doping, electrons drift towards the GaN NW core, while holes drift towards the HOPG substrate. The reverse carrier flow takes place for p-type doping. In both cases this results is a NW Fermi level shift (with respect to the grounded HOPG Fermi level) that is upwards for n-type and downwards for p-type. This behavior accounts for the photogenerated voltage that amounts to $\mathrm{V}_{\mathrm{ph}}=340 \mathrm{mV}$ for both types of doping. The opposite shift of the Fermi level under illumination further confirms the n- and p-type character of the studied NWs. Since the photogenerated voltage at the NW/HOPG contact has the same sign as that generated at the p-n junction, the photovoltage measured at the p-n junction $(\sim 1.2 \mathrm{~V})$ is the result of the combined effect of the junction and the Schottky contacts at the $\mathrm{n}$ and $\mathrm{p}$ sides. 
Additional information about carrier dynamics has been obtained by analyzing the time evolution of the surface photovoltage (SPV), defined as the difference of $\mathrm{V}_{\mathrm{CPD}}$ under illumination and in dark. The results are represented in Figure 8. The evolution of SPV when light is switched on and off is very different in both samples. In the p-type NW [panel b)] illumination causes a sudden upward jump in SPV, followed by a gradual decrease. When switching-off the illumination the initial values $(\mathrm{t}=0)$ are also recovered very quickly. Instead, illumination of the $\mathrm{n}$-doped NW [panel a)] causes a slower decrease of the SPV values, while SPV increases in dark after illumination at an even slower rate. In order to quantify these changes, following Ref. [29] the data have been fitted with stretched exponential functions (full line in Figure 8). A single function was enough to describe the rise and decay of SPV for the n-type NW. In this case, the decay time characterizing the SPV drop upon illumination is in the range of tens of seconds ( $\tau=12 \mathrm{~s}$ ), similarly as in Ref. [29] for n-type c-plane samples. The rising of SPV in dark after sample illumination is about ten times slower ( $\tau=107 \mathrm{~s})$. Interestingly, SPV changes in the p-type NW are drastically shorter, occurring in less than $0.4 \mathrm{~s}$, which is the resolution of our measurements. Attempts to fit the experimental data lead to rise time values upon illumination of the order of $0.01 \mathrm{~s}$, and subsequent slower decay values in dark of $\sim 0.2$ s. Besides this, we observe a steady decrease of SPV during illumination, a characteristic that, as commented before, has been ascribed to the photoinduced chemisorption of oxygen [23]. The transfer of photo-generated electrons to the adsorbed oxygen species results in gradually decreasing $\mathrm{V}_{\mathrm{CPD}}$. Such a gradual $\mathrm{V}_{\mathrm{CPD}}$ decrease under illumination is also observed in the n-type NW but with a faster rate, pointing to a higher oxygen chemisorption in the Si doped NW. The fact that this decrease is not observed in the p-n junction will be the subject of future investigations, but points to the large variability of surface effects, that strongly depend on the energy of the surface states and the previous history of the sample.

The different dynamics observed for $\mathrm{n}$ - and p-type NWs is tentatively assigned to one or a combination of the following contributions, namely: i) the role of surface states or dopant on 
surface reactivity with physisorbed oxygen, ii) the different surface oxide thickness for $\mathrm{p}$ - and $\mathrm{n}$ type NWs, limiting the charge transfer between the NW and the physisorbed oxygen.

Discrimination between these contributions would require SPV measurements in well-controlled environments $\left(\mathrm{N}_{2}\right.$ and $\left.\mathrm{O}_{2}\right)[23]$ and oxide-free samples.
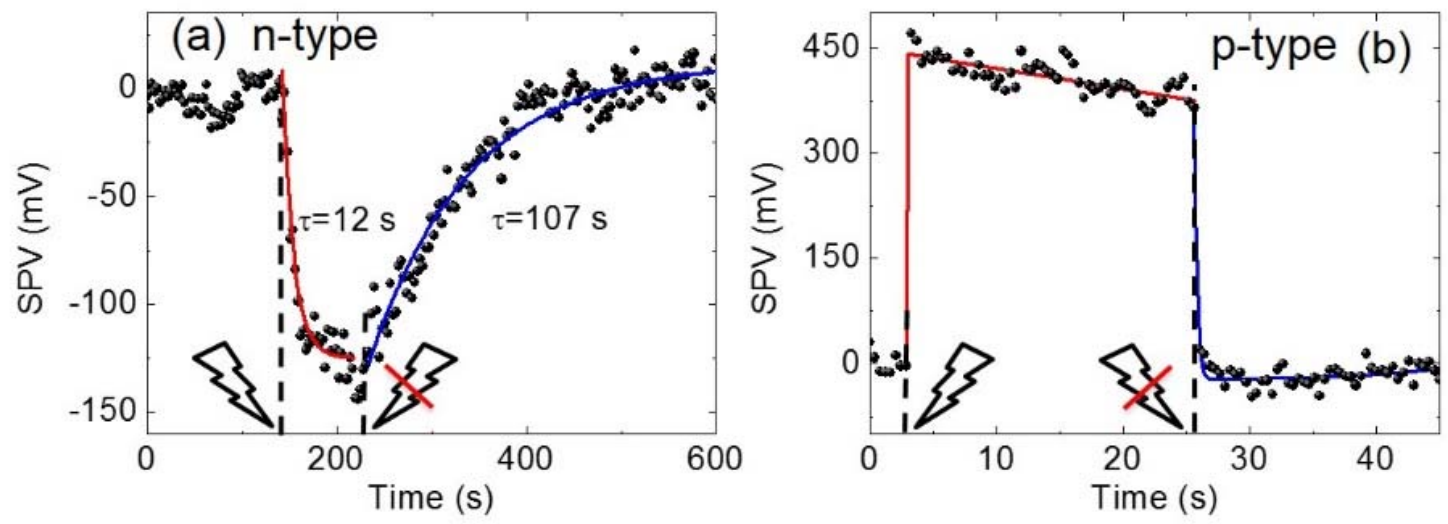

Figure 8: Evolution of the SPV of a) n-type and b) p-type NWs with time after illumination with UV light.

\section{Conclusion}

In conclusion, it has to be reminded that being able to identify the spatial location of the p-n junction and its basic electrical characteristics is a prerequisite for controlling the electro-optical properties of NW-based devices. Along these lines, KPFM has allowed us to directly study the characteristics of several p-n junctions in GaN single NWs, in terms of built-in potential and depletion width. The existence of a p-n junction in the GaN NWs has been demonstrated by the change in $\mathrm{V}_{\mathrm{CPD}}$ from the $\mathrm{n}$ - to the $\mathrm{p}$-side, which for NWs deposited on HOPG, varies from 0.17 to $1.59 \mathrm{~V}$ depending on NW diameter. 3D finite element simulations have been performed, leading to a satisfactory agreement with the experimental data, although the uncertainty in the nature of surface states hinders a more detailed quantification of the experimental results. Nonetheless, we would like to stand out that our measurements can be extended to the study of the open circuit voltage under illumination without further contact processing. Indeed, under the experimental configuration discussed here, the shift of 
the Fermi levels at the $\mathrm{n}$ and $\mathrm{p}$ sides of the junction upon UV illumination could be probed, obtaining a photovoltage of $1.2 \mathrm{~V}$. The photovoltage of the NW/HOPG Schottky contact has been studied by analyzing the $\mathrm{V}_{\mathrm{CPD}}$ and SPV of NW segments. The p- or n-type doping of these segments can be clearly distinguished by their different $\mathrm{V}_{\mathrm{CPD}}$, as well as by their characteristic SPV behavior. However, quantification of doping would require a more detailed study of the surface band-bending. The time evolution of the SPV points to a higher oxygen chemisorption at the n-type NW than at the p-type. As a whole, we have found that the shift of the Fermi-level induced by the photogenerated carriers, and not the decrease of band bending, is the main effect of UV illumination in our samples. Finally, it has been demonstrated that the observed p-n junction photovoltage arises from the combined effect of the junction and the NW/HOPG Schottky contact, which contributes by around $340 \mathrm{mV}$ for separated $\mathrm{p}$ - and n-type segments.

\section{Experimental Section}

Growth of GaN NW p-n junction: GaN NWs were grown along the c-axis of the wurtzite structure by molecular beam epitaxy in a MECA2000 reactor on a 2-inch (111) Si wafer. Prior to NW growth a thin AlN buffer layer was deposited in order to improve nanowire orientation [30]. The base was grown at a substrate temperature of $860^{\circ} \mathrm{C}$ and $\mathrm{n}$-doping was achieved using $\mathrm{Si}$ (the $\mathrm{Si}$ effusion cell temperature was $875^{\circ} \mathrm{C}$ ). For the upper part of the NWs the substrate temperature was lowered to $775{ }^{\circ} \mathrm{C}$ and p-type doping was obtained using $\mathrm{Mg}$ (Mg effusion cell temperature of $220^{\circ} \mathrm{C}$ ). The carrier concentration of the n-type region was evaluated by four probe measurements performed on single Si-doped GaN NWs grown under similar conditions, leading to a free electron density in the range of $10^{18} \mathrm{~cm}^{-3}[31]$. Mg incorporation in the p-type region $\left([\mathrm{Mg}]=3.4 \times 10^{19} \mathrm{~cm}^{-3}\right)$ was determined by energy dispersive x-ray spectroscopy measurements of GaN NWs grown under similar conditions, corresponding to a higher limit for doping level (active $\mathrm{Mg}$ acceptor density $\mathrm{N}_{\mathrm{A}} \leq 3.4 \times 10^{19} \mathrm{~cm}^{-3}$ ).

Electrical characterization. KPFM and SPV: A NanoTec atomic force microscope was used for KPFM. The system is equipped with a phase-locked loopboard (PLL, bandwidth $\sim 2 \mathrm{kHz}$ ) which 
maintained the cantilever at resonance. Pt coated cantilevers (HQ:NSC14/Pt from $\mu$-Masch, $4.5 \mathrm{~N} / \mathrm{m}$ force constant, resonance frequency $\sim 140 \mathrm{kHz}$ ) were used for the experiment. Topography and KPFM were acquired using the oscillation amplitude as feedback parameter (AM-mode) in a single-pass mode. Measurement of the contact potential difference $\left(\mathrm{V}_{\mathrm{CPD}}\right)$ between tip and sample proceeded by applying a fixed $\mathrm{AC}$ voltage $(0.7 \mathrm{~V})$ at $7 \mathrm{kHz}$ to the $\mathrm{AFM}$ tip while the sample was held at ground potential. For small amplitudes in the true non-contact regime, and provided that the AC frequency is much smaller than the tip resonance frequency, the shift of the resonance frequency is proportional to the gradient of the electrostatic interaction [32]. A second feedback adjusts the DC bias between tip and sample in order to minimize this interaction and determine $\mathrm{V}_{\mathrm{CPD}}$ (FM-mode). Consequently, the measurement is performed in the so-called AM-FM mode [33]. Tip-sample distance was maintained between 5 and $10 \mathrm{~nm}$, resulting in $\mathrm{V}_{\mathrm{CPD}}$ imaging with high spatial $(\sim 10 \mathrm{~nm})$ and potential $(\sim 30 \mathrm{~V})$ resolution $[32,34]$. For SPV the samples were illuminated from the top by the light of a 100 W Oriel mercury lamp with the use of an optical fiber. At the exit of the fiber, the light was collimated and focused on the sample by a quartz lens with $10 \mathrm{~mm}$ focal length, resulting in a spot of $\sim 1 \mathrm{~cm}^{2}$. Quantitative comparison of CPD between NWs has been performed by taking the surface potential of the HOPG substrate as reference.

$3 D$ finite element simulation: Electrostatic properties of the p-n junction GaN NWs have been simulated by solving Poisson's equation, thanks to 3D finite element software (nextnano3). We assumed a circular GaN wurtzite NW (50 nm diameter and $2000 \mathrm{~nm}$ length) with a c-growth axis along the horizontal $\mathrm{x}$-direction and an abrupt p-n junction at half-length of the NW. The NW is surrounded by air. On Fig 3, the $x, y, z$ origin of the position is taken at the intercept between the p-n junction plane and the circular axis of the NW. The figure illustrates the electrostatic properties of the NW's core showing a $x y$ cross section $(z=0)$, which corresponds to a $2 \mathrm{D}$ slice belonging the circular axis. A similar doping level has been assumed at each side of the $p-n$ junction, i.e. $\mathrm{N}_{D}=\mathrm{N}_{A}=3.8 \times 10^{18}$ 
$\mathrm{cm}^{-3}$. Negative (positive) fixed surface charges have been considered as acceptor (donor) on $\mathrm{n}-(\mathrm{p}-)$ side of the wire with an surface density of $\mathrm{N}_{\text {surf }}=3 \times 10^{12} \mathrm{~cm}^{-2}$.

\section{Supporting Information}

Supporting Information is available.

\section{Acknowledgements}

This work has been financed by the European ITN PITN-GA-2010-265073 Nanowiring

\section{References}

[1] S. Nakamura, S. Pearton, G. Fasol in The Blue Diode. The Complete Story. Springer-Verlag Berlin Heidelberg 2000.

[2] K. Jeganathan, R. K. Debnath, R. Meijers, T. Stoica, R. Calarco, D. Grützmacher, H. Lüth, J. Appl. Phys. 2009, 105, 123707.

[3] D. Lindgren, O. Hultin, M. Heurlin, K. Storm, M. T. Borgström, L. Samuelson, A. Gustafsson, Nanotechnology 2015, 26, 045705.

[4] P. Tchoulfian, F. Donatini, F. Levy, A. Dussaigne, P. Ferret, J. Pernot, Nano Lett. 2014, 14, 3491.

[5] S. Kalinin, A. Gruverman in Scanning Probe Microscopy. Electrical and Electromechanical Phenomena at the Nanoscale. Springer-Verlag Berlin Heidelberg 2007.

[6] S. Vinaji, A. Lochthofen, W. Mertin, I. Regolin, C. Gutsche, W. Prost, F. J. Tegude, G. Bacher, Nanotechnology 2009, 20, 385702.

[7] A. C. Narváez, T. Chiaramonte, K. O. Vicaro, J. H. Clerici, M. Cotta, Nanotechnology 2009, 20, 465705 .

[8] N. J. Quitoriano, R. N. Sanderson, S.-S. Bae, R. Ragan, Nanotechnology 2013, 24, 205704.

[9] R. Nowak, D. Moraru, T. Mizuno, R. Jablonski, M. Tabe, Appl. Phys. Lett. 2013, 102, 083109.

[10] R. Nowak, D. Moraru, T. Mizuno, R. Jablonski, M. Tabe, Thin Solid Films 2014, 557, 249. 
[11] A. Imtiaz, T. M. Wallis, J. C. Weber, K. J. Coakley, M. D. Brubaker, P. T. Blanchard, K. A. Bertness, N. A. Sanford, P. Kabos, Appl. Phys. Lett. 2014, 104, 263107.

[12] R. Mata, K. Hestroffer, J. Budagosky, A. Cros, C. Bougerol, H. Renevier, B. Daudin, J. Cryst. Growth 2011, 337, 177.

[13] W. N. Hansen, G. J. Hansen, Surf. Sci. 2001, 481, 172.

[14] C. Y. Nam, D. Tham, J. E. Fischer, Nano Lett. 2005, 5, 2029.

[15] C.-L. Tsai, Y.-J Lin, J.-H. Lin, J. Mater. Sci.: Mater. Electron. 2015, 26, 3052.

[16] S. Kim, F. Shafiei, D. Ratchford, X. Li, Nanotechnology 2011, 22, 115301.

[17] R. Shikler, T. Meoded, N. Fried, B. Mishori, Y. Rosenwaks, Appl. Phys. Lett. 1999, 86, 107.

[18] R. Calarco, M. Marso, T. Richter, A.I. Aykanat, R. Meijers, A.v.d. Hart, T. Stoica, H. Lüth, Nano Lett. 2005, 5, 981.

[19] J. P. Long, V. M. Bermudez, Phys. Rev. B 2002, 66, 121308.

[20] Here, all the fixed charges at the surface are considered ionized because they are induced by a trap level located in a band (conduction band for the donor on the p-type surface and valence band for the acceptor in the n-type surface).

[21] J. D. Wei, S. F. Li, A. Atamuratov, H.-H. Wehmann, A. Waag, Appl. Phys. Lett. 2010, 97, 172111.

[22] M. Foussekis, A. A. Baski, M. A. Reshchikov, Appl. Phys. Lett. 2009, 94, 162116.

[23] M. Foussekis, A. A. Baski, M. A. Reshchikov, J. Vac. Sci. Technol. B 2011, 29, 041205.

[24] T. Stoica, E. Sutter, R. Calarco, in Trends in Nanophysics, Engineering Materials, Edited by A. Aldea, V. Bârsan. Springer-Verlag Berlin Heidelberg 2010.

[25] M.G. Kibria, S. Zhao, F.A. Chowdhury, Q. Wang, H.P.T. Nguyen, M.L. Trudeau, H. Guo, Z. Mi, Nat. Commun. 2013, 5, 3825.

[26] T. Hashizume, J. Appl. Phys. 2003, 94, 431.

[27] S. Barbet, R. Aubry, M.-A. di Forte-Poisson, J.-C. Jacquet, D. Deresmes, T. Mélin, D. Théron, Appl. Phys. Lett. 2008, 93, 212107. 
[28] L. Kronik, Y. Shapira, Surf. Sci. Rep. 1999, 37, 1.

[29] A. Winnerl, R. N. Pereira, M. Stutzmann, Phys. Rev. B 2015, 91, 075316.

[30] R. Songmuang, O. Landre, B. Daudin, Appl. Phys. Lett. 2007, 91, 251902.

[31] Z. Fang, E. Robin, E. Rozas-Jiménez, A. Cros, F. Donatini, N. Mollard, J. Pernot, B. Daudin, Si donor incorporation in GaN nanowires. Nano Lett. 2015, 15, 6794.

[32] J. Colchero, A. I. Gil, A. M. Baró, Phys. Rev. B 2001, 64, 245403.

[33] Advancing Characterization of Materials with Atomic Force Microscopy-Based Electric Techniques. S. Magonov, J. Alexander, S. Wu. Chapter 9 in Scanning Probe Microscopy of Functional Materials. Nanoscale Imaging and Spectroscopy. S. V. Kalinin and A. Gruverman Eds. 2010 Springer.

[34] E. Placios-Lidón, J. Abellán, C. Munuera, C. Ocal, J. Colchero, Appl. Phys. Lett. 2005, 87, 154106. 